\title{
The Effect of Prophylactic Central Neck Dissection During Hemithyroidectomy on Locoregional Recurrence in Patients With Papillary Thyroid Carcinoma: A Meta-Analysis
}

\author{
Soon-Hyun Ahn (iD · Won Sik Kim (i) \\ Department of Otorhinolaryngology-Head and Neck Surgery, Seoul National University Hospital, \\ Seoul National University College of Medicine, Seoul, Korea
}

Objectives. Hemithyroidectomy is commonly performed in patients with low- to intermediate-risk papillary thyroid carcinoma. The purpose of this meta-analysis was to evaluate the effect of prophylactic central neck dissection on locoregional recurrence in patients undergoing hemithyroidectomy.

Methods. A meta-analysis was performed of full-text publications published in English retrieved from the Embase database.

Results. The rate of regional recurrence in the central compartment after hemithyroidectomy, with or without prophylactic central neck dissection, was $0.17 \%$ and $1.78 \%$, respectively. This difference was statistically significant. Recurrence in the lateral compartment or contralateral thyroid was not affected by prophylactic central neck dissection; the overall rate of recurrence was $1.3 \%$ and $5.4 \%$, respectively.

Conclusion. Prophylactic central neck dissection significantly reduced the risk of recurrence in the central compartment in patients undergoing hemithyroidectomy.

Keywords. Central Neck Dissection; Locoregional Recurrence; Papillary Thyroid Carcinoma; Thyroidectomy; Lymph Node

\section{INTRODUCTION}

In light of recent articles that have reported no significant differences in oncological results between hemithyroidectomy and total thyroidectomy in patients with low- to intermediate-risk papillary thyroid carcinoma, the indications for hemithyroidectomy have been expanded [1,2]. However, controversy regarding central neck dissection persists, even in cases of hemithyroidectomy, given that occult nodal metastasis in the central compartment has been observed in approximately one-half of patients clinically diagnosed with N0 papillary carcinoma [3,4]. Some meta-analyses have investigated the effectiveness of prophylactic central neck dissection performed with total thyroidectomy [5-7], but not in patients undergoing hemithyroidectomy alone. The purpose of this study, therefore, was to evaluate the oncological effect of prophylactic central neck dissection performed during hemithyroidectomy in patients with low- to intermediate-risk papillary thyroid carcinoma.

\section{MATERIALS AND METHODS}

Literature search

The Embase and Medline database was searched on June 3, 2019 using the terms "papillary thyroid carcinoma," "hemithyroidectomy," "lobectomy," and "central OR node."

Copyright (C) 2020 by Korean Society of Otorhinolaryngology-Head and Neck Surgery.

This is an open-access article distributed under the terms of the Creative Commons Attribution Non-Commercial License (https://creativecommons.org/licenses/by-nc/4.0)

which permits unrestricted non-commercial use, distribution, and reproduction in any medium, provided the original work is properly cited. 


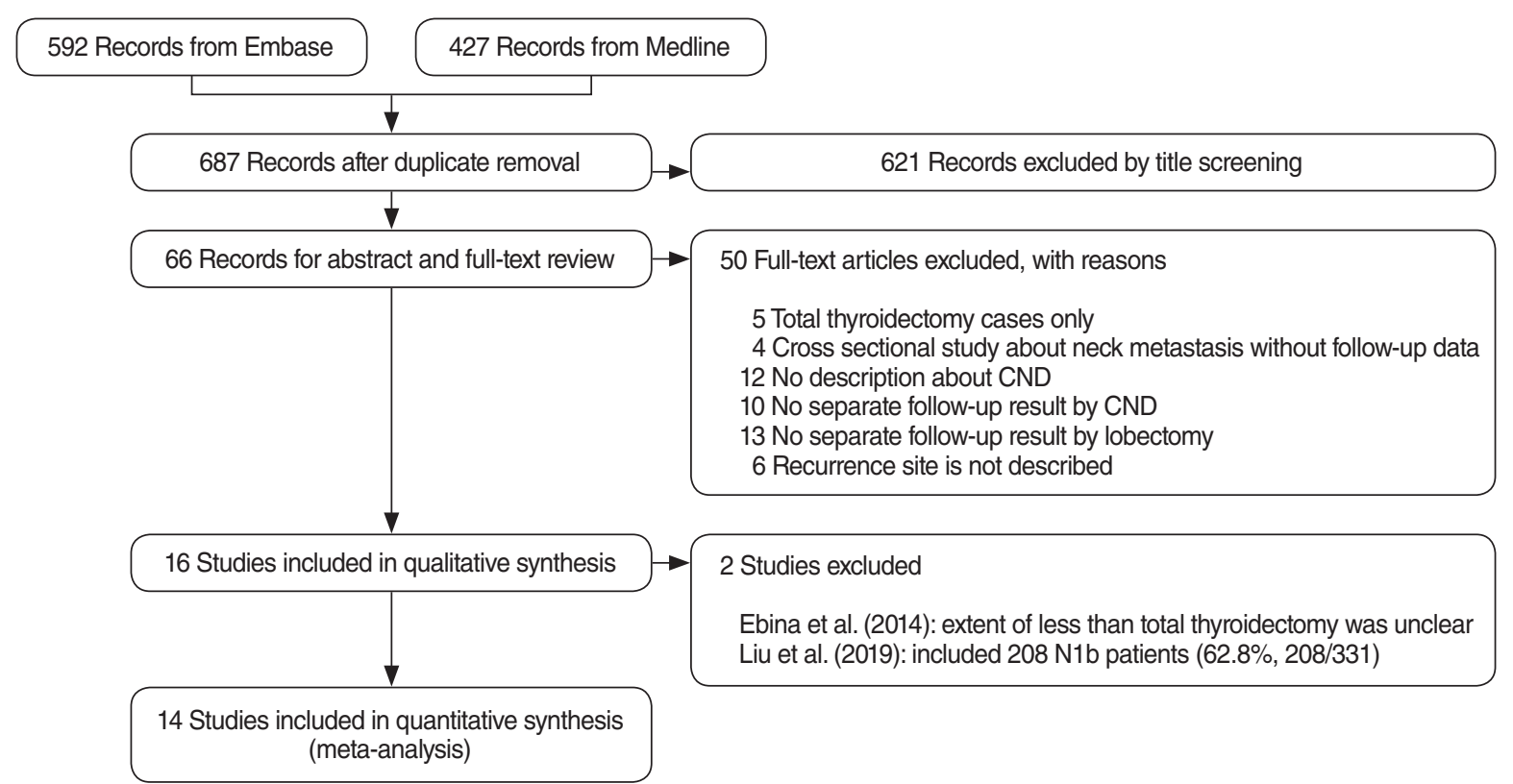

Fig. 1. Flow diagram illustrating the literature search protocol. CND, central neck dissection.

\section{Inclusion criteria}

Observational studies published as full-text articles in English were screened using following inclusion criteria: performance of central neck dissection was clearly stated; extent of thyroidectomy was clearly defined, and data regarding hemithyroidectomy were separately reported; recurrence was reported separately according to site (i.e., contralateral thyroid, and central and lateral compartments); and studies included patients with low to intermediate risk papillary thyroid carcinoma.

\section{Data analysis}

There were only two comparative studies; as such, all observational studies reporting oncological results after hemithyroidectomy-with or without central neck dissection-were searched and underwent proportional meta-analysis. The recurrence rate was calculated for the contralateral thyroid, and central and lateral compartments, separately. The proportional meta-analysis was performed using StatsDirect Ver. 3 (Cheshire, UK) and comprehensive meta-analysis (Biostat, Englewood, NJ, USA). Forest

\section{H I G G H L I $G$ G H T T S}

- Recurrence in the central compartment was lower in the central neck dissection (CND) group.

- The central compartment recurrence rate was $0.17 \%$ with CND and $1.78 \%$ without CND.

- The lateral compartment recurrence rate was $1.3 \%$, with no difference according to whether CND was performed.

- New carcinoma in the contralateral lobe was observed in 5.4\% of cases overall. plots were constructed using Excel (Microsoft Corp., Redmond, WA, USA). Quality assessment of the included articles was performed using the risk of bias assessment tool for non-randomized studies (RoBNAS) and Review Manager ver. 5.3 (Cochrane Collaboration, Copenhagen, Denmark).

\section{RESULTS}

In total, 687 records were retrieved from the Embase database after removal of duplicates. Upon screening article titles, 621 irrelevant records were excluded. The abstract and full text of 66 records were screened, and 14 were included in the quantitative analysis. The articles published by Ebina et al. [8] and Liu et al. [9] reported follow-up data for patients who underwent hemithyroidectomy with central neck dissection, but were excluded. In the study by Ebina et al. [8], the extent of hemithyroidectomy was unclear and many subtotal thyroidectomies were included. In the study by Liu et al. [9], $62.8 \%$ of patients exhibited lateral neck metastasis, and it appeared that the researchers applied different indications from what is generally accepted (Fig. 1).

Table 1 summarizes the outcomes of the 14 studies that were ultimately included in the analysis [10-23]. Two studies investigated the impact of prophylactic central neck dissection in hemithyroidectomy using a comparative method [11,19]. Six investigations compared oncological results between total thyroidectomy and hemithyroidectomy in various subjects; however, only the data regarding hemithyroidectomy were included in the present study $[10,12,13,20,21,23]$. Others compared recurrence according to various factors among patients who underwent hemithyroidectomy [14-18,22]. The patients included were 


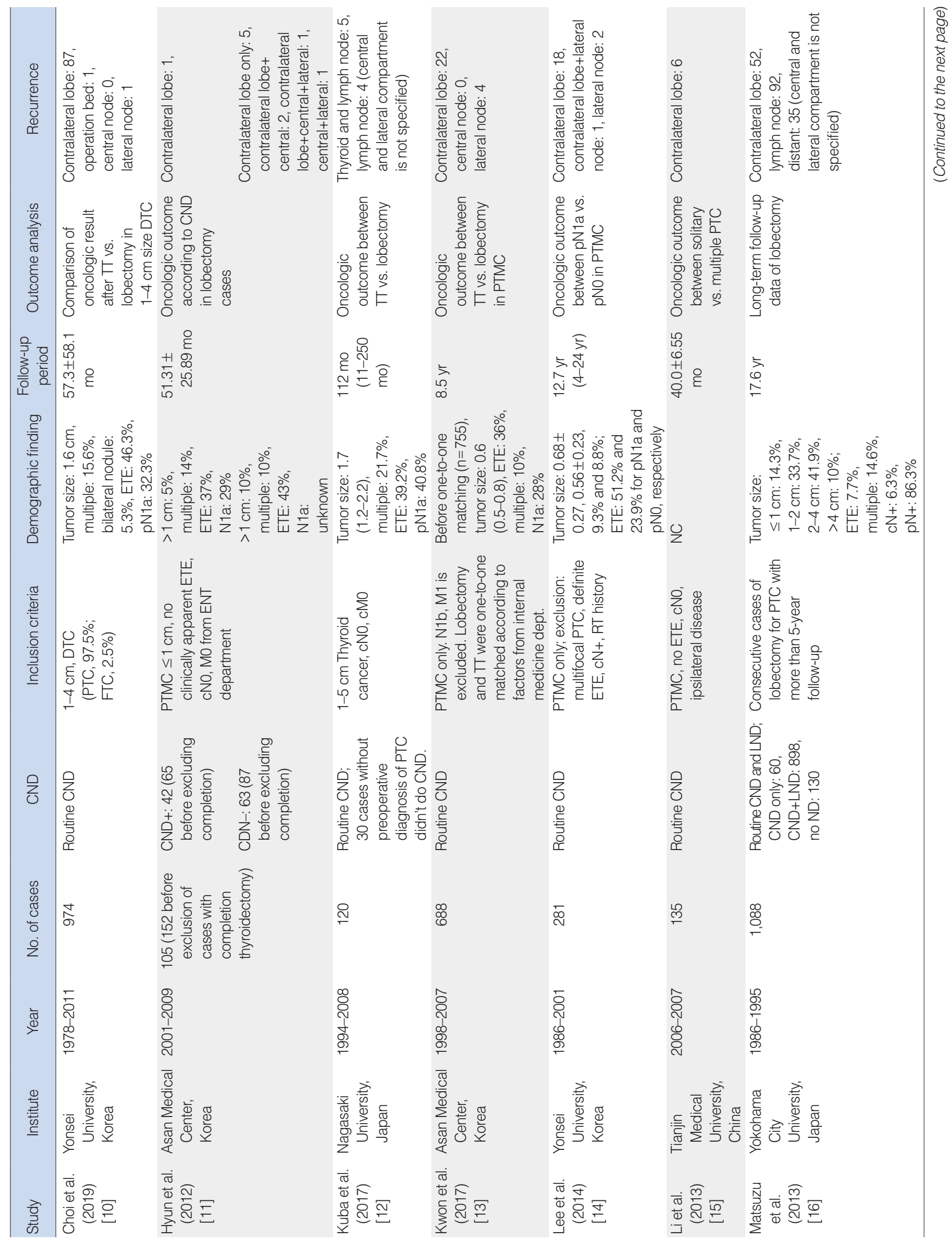




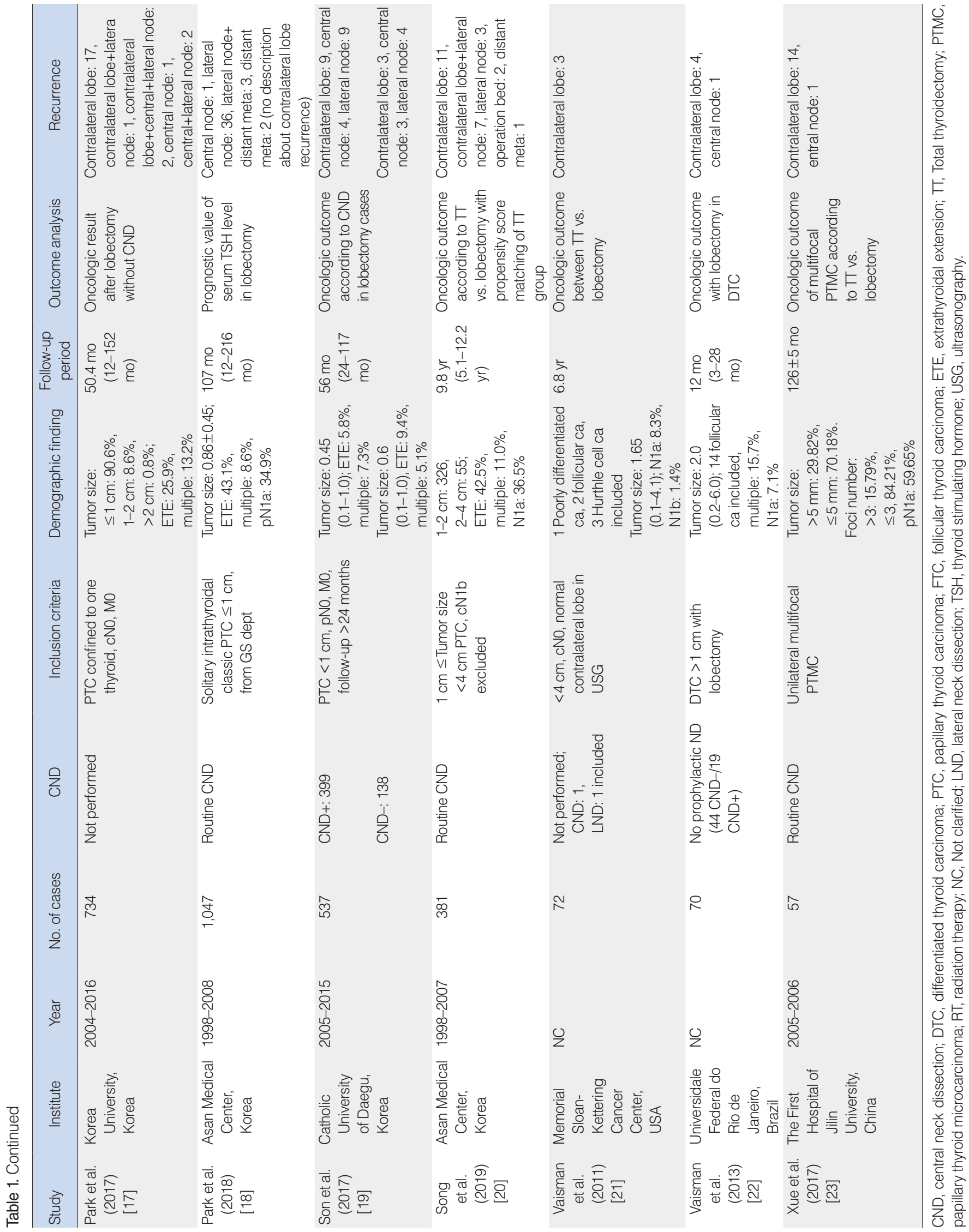


mostly those with a tumor size $<4 \mathrm{~cm}$ and no clinical preoperative lymph node metastasis. The rates of developing new carcinoma in the contralateral thyroid, lymph node metastasis in the central compartment, and lymph node metastasis in the lateral compartment were calculated separately. In studies in which routine central neck dissection was performed, the rate of occult metastasis in the central compartment was found to range from $28.0 \%$ to $59.65 \%$.

In the quality assessment according to the RoBNAS criteria, most studies were found to be free of problems. Kwon et al. [13] did not report oncological results from all patients; results were only reported for 688 of the 755 patients used for case-control

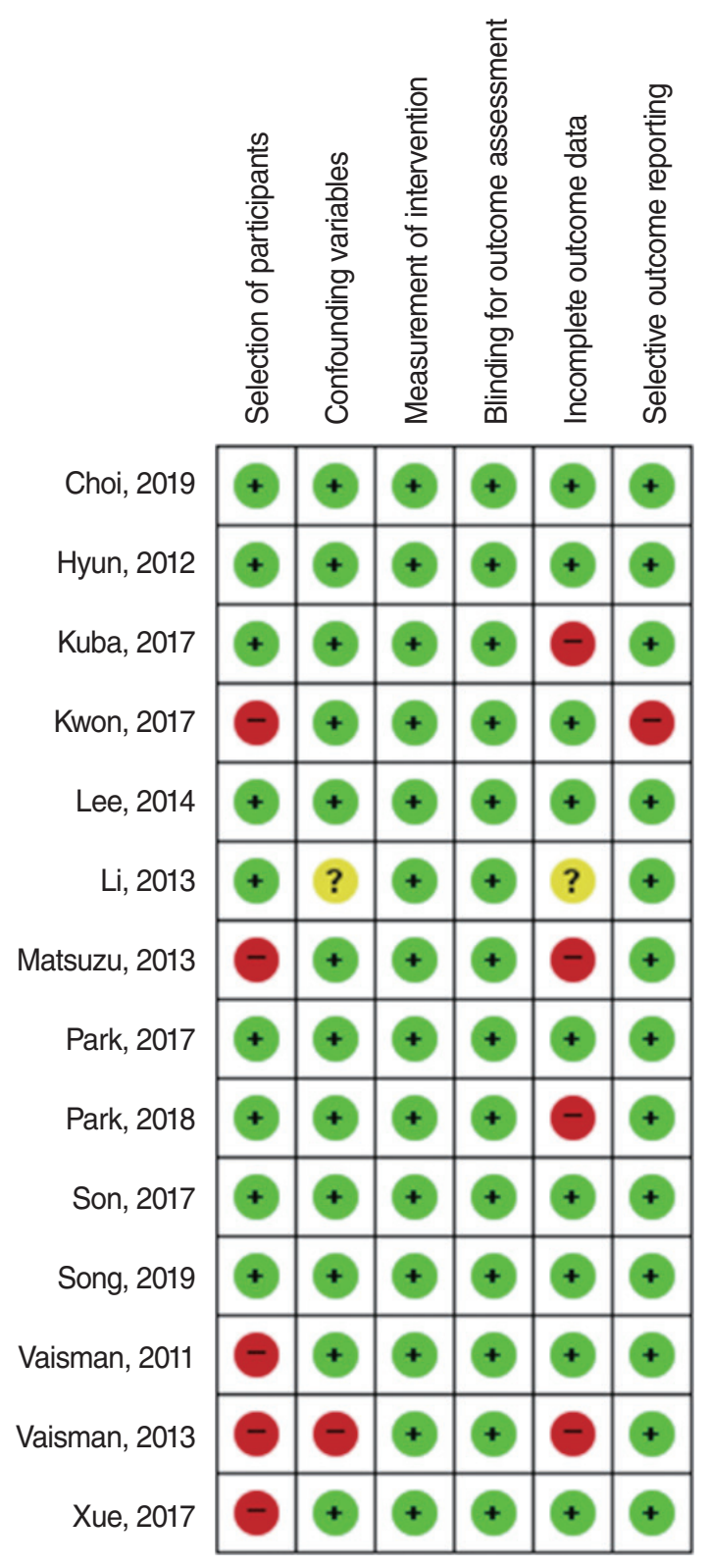

Fig. 2. Risk of bias summary according to the risk of bias assessment tool for non-randomized studies. matching. Vaisman et al. [21,22] included cases other than papillary carcinoma in both studies. Xue et al. [23] only selected cases of multifocal papillary thyroid carcinoma. Therefore, these four studies were classified as having a high risk of bias in participant selection. Vaisman et al. [22] did not clearly describe the number of patients who underwent central neck dissection, although the authors reported that they did not routinely perform prophylactic central neck dissection; therefore, their study was classified as having a high risk of bias in confounding variables. Studies by Kuba et al. [12] and Matsuzu et al. [16] did not report lymph node metastasis in the central and lateral compartments separately. Park et al. [18] did not report recurrence in the contralateral thyroid and, in one study by Vaisman et al. [22], the follow-up period was too short (12 months; range, 3-28 months), making it difficult to calculate the true recurrence rate. Thus, these studies were classified as having a high risk for bias in incomplete outcome data (Fig. 2).

In the proportional meta-analysis, central compartment recurrence occurred in $0 \%$ to $1.75 \%$ of cases, with a mean of $0.17 \%$ when prophylactic central neck dissection was performed. When it was not performed, the recurrence rate was found to be $0 \%$ to $6.4 \%$, with a mean of $1.78 \%$. A between-group comparison according to whether prophylactic central neck dissection was performed yielded a significant difference $(P=0.018)$ (Fig. 3A). Lateral compartment recurrence demonstrated no difference according to whether prophylactic central neck dissection was performed; the recurrence rate ranged from $0 \%$ to $3.72 \%$, with a mean of $1.31 \%$ (Fig. 3B). Carcinoma in the contralateral thyroid was observed in $2.17 \%$ to $24.6 \%$ of cases, with a mean of $5.44 \%$ in total; no significant difference was found according to whether central neck dissection was performed (Fig. 3C).

\section{DISCUSSION}

Although many studies have investigated the issue, controversy continues regarding the value of prophylactic central neck dissection. In the American Thyroid Association (ATA) guidelines published in 2016, prophylactic central neck dissection was not recommended for T1- or T2-differentiated thyroid cancers [24]. The authors of the guideline stated that prophylactic central neck dissection can identify a substantial number of pN1 patients; however, the direct effect of this procedure is small. Another problem with central neck dissection during total thyroidectomy is that morbidity can be excessively high when an inexperienced surgeon is operating.

Some meta-analyses have analyzed the effect of central neck dissection in patients undergoing total thyroidectomy. A common finding of those studies is that prophylactic central neck dissection reduced locoregional recurrence from $4.59 \%-8.6 \%$ to $2.52 \%-5.9 \%[5-7,25]$. This change in locoregional recurrence was due to decreased recurrence in the central compartment, 


\section{Recurrence in Central Compartment}

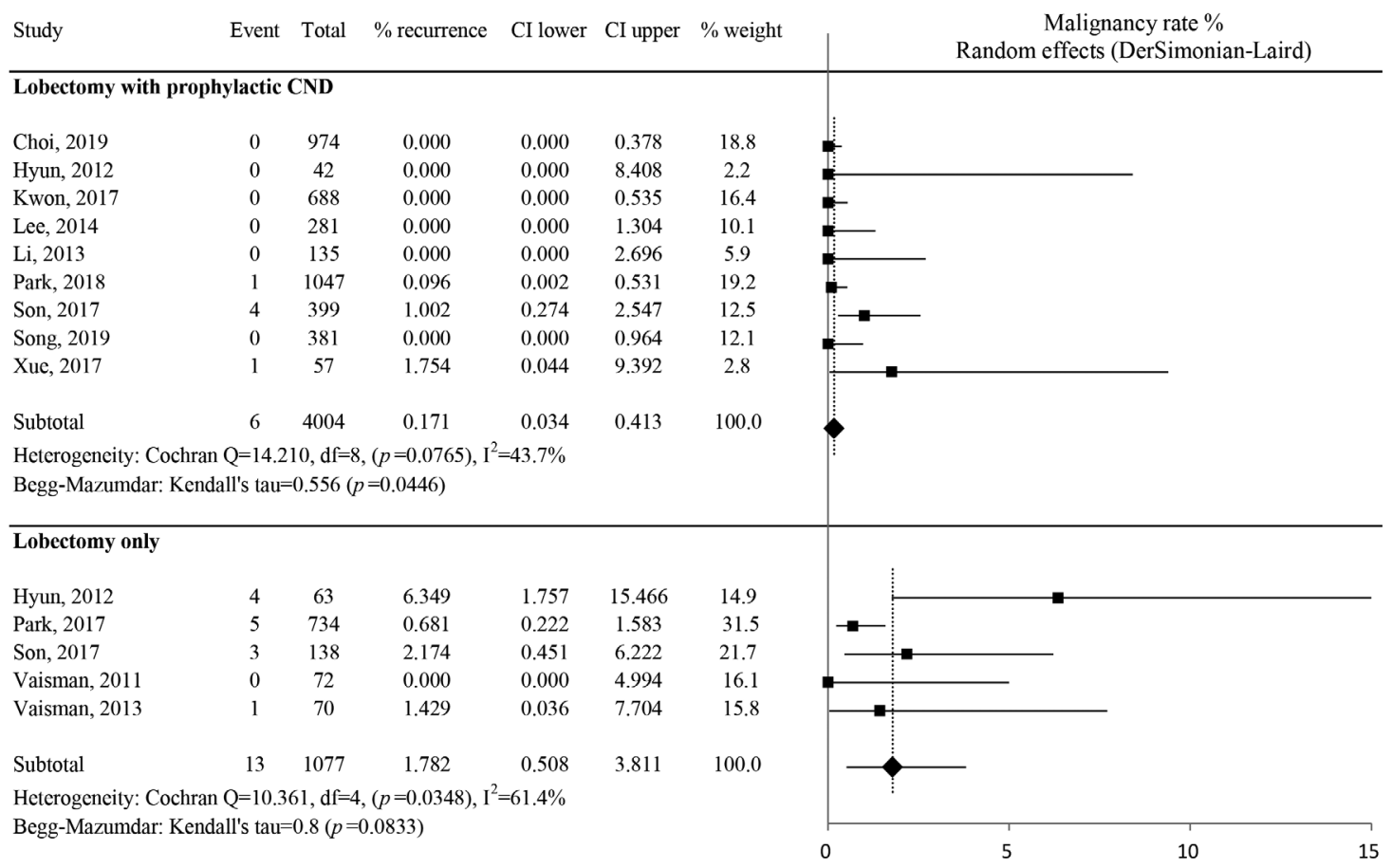

Total between: Cochrane $Q=5.590, \mathrm{df}=1,(p=0.018)$

$\begin{array}{llllll}\text { Overall } & 19 & 5081 & 0.487 & 0.172 & 0.963\end{array}$

Recurrence in Lateral Compartment

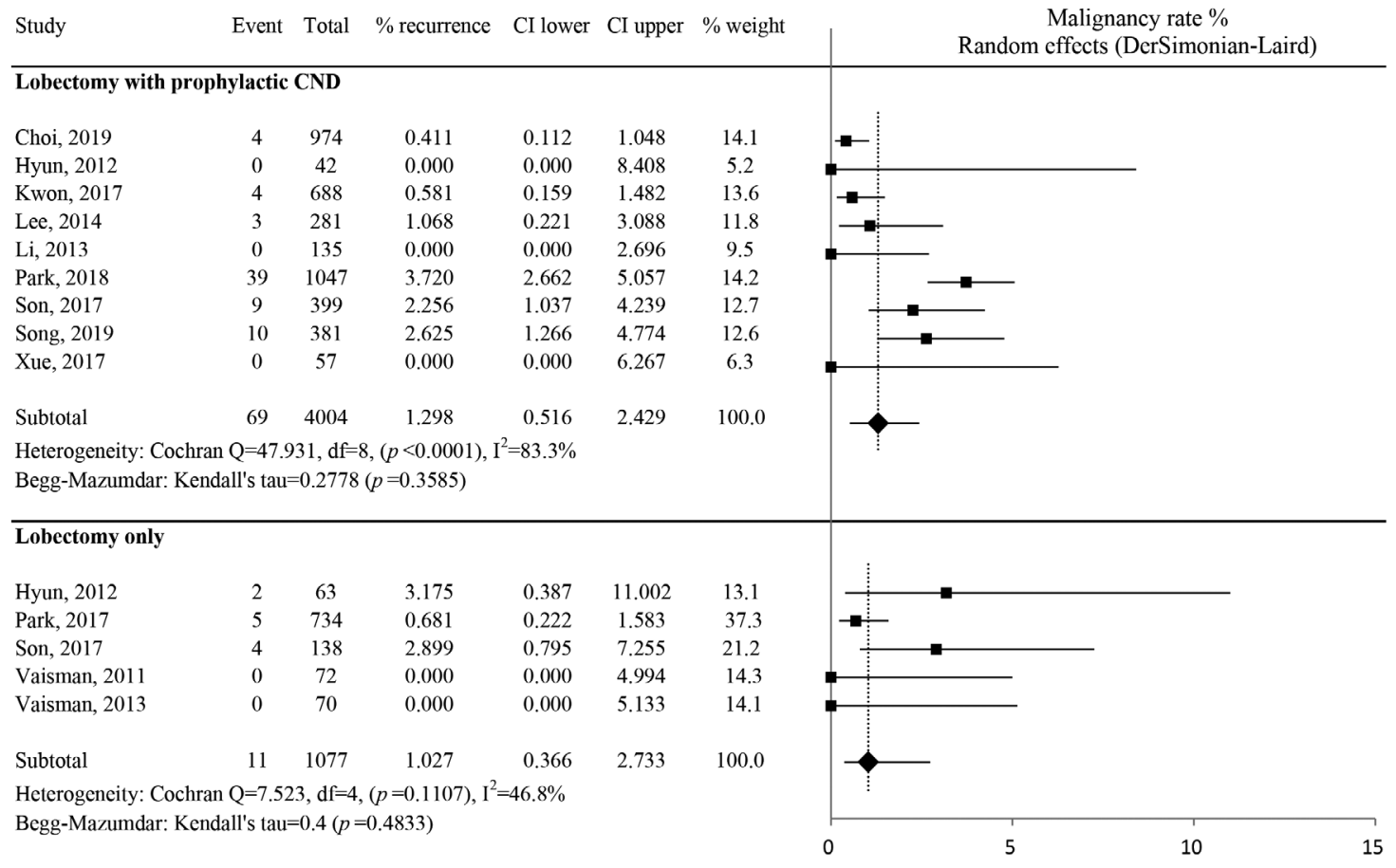

Total between: Cochrane $\mathrm{Q}=0.0027, \mathrm{df}=1,(p=0.959)$

$\begin{array}{lllllllll}\text { Overall } & 80 & 5081 & 1.312 & 0.671 & 2.163 & \text { B }\end{array}$

Fig. 3. Proportional analysis of recurrence after lobectomy with or without central neck dissection. Comparisons of recurrence in the central compartment $(A)$, recurrence in the lateral compartment $(B)$. Cl, confidence interval; $C N D$, central neck dissection. (Continued to the next page) 
Recurrence in Contralateral Thyroid

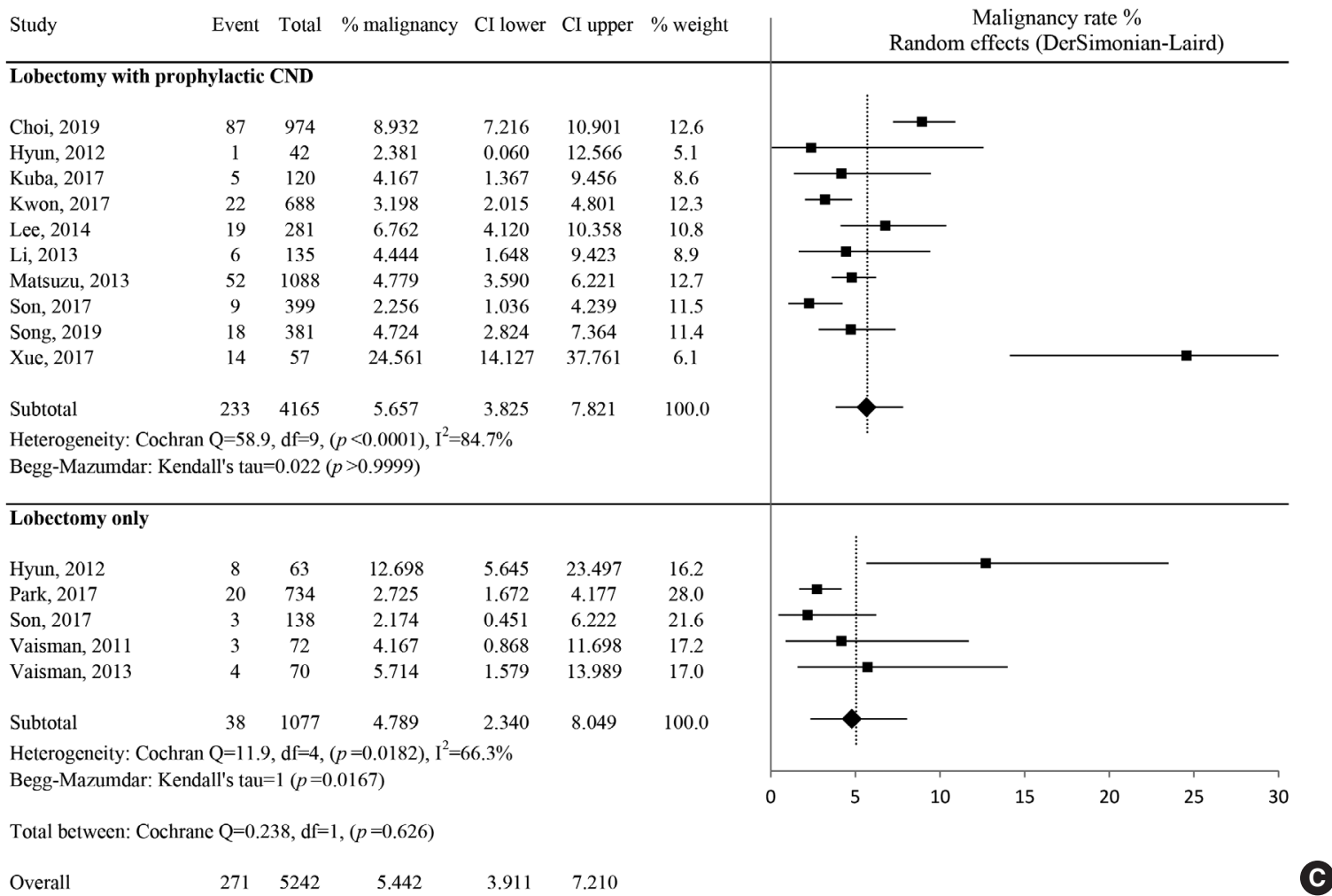

Fig. 3. (Continued) Proportional analysis of recurrence after lobectomy with or without central neck dissection. (C) Comparison of recurrence in the contralateral thyroid. $\mathrm{Cl}$, confidence interval; CND, central neck dissection.

but not in the lateral compartment [7]. However, radioactive iodine therapy and thyroid-stimulating hormone (TSH) suppression can be a confounding factor for this reduction in regional recurrence rates. When a tumor is pathologically diagnosed as $\mathrm{N} 1$ by central neck dissection, radioactive iodine therapy may be used with a higher dose and more stringent TSH suppression will be applied. Therefore, although there is no clear evidence that the addition of radioactive iodine therapy or TSH suppression reduces recurrence, postoperative treatment could be a confounding variable. In contrast, morbidity is significantly increased by central neck dissection in patients undergoing total thyroidectomy. According to previous meta-analyses, the risk for recurrent laryngeal nerve paralysis did not change according to whether central neck dissection was performed. However, central neck dissection significantly increased the risk of transient and permanent hypocalcemia [5-7]. Therefore, balancing the risks and benefits of central neck dissection is an important consideration in patients undergoing total thyroidectomy.

Until the revised ATA guideline was published in 2016, total thyroidectomy was regarded as the optimal surgical procedure in most cases of papillary thyroid carcinoma. However, recent reports describing outcomes in patients receiving active surveillance for papillary microcarcinoma have significantly changed surgeons' approach to thyroid surgery [26,27], and hemithyroid- ectomy-as a more conservative procedure-is now regarded as optimal in many instances, even in the revised ATA guidelines [24]. Nevertheless no meta-analyses have yet investigated the effects of central neck dissection in hemithyroidectomy. In addition, radioactive iodine therapy is not possible after hemithyroidectomy, and TSH suppression is not applied or only minimally applied. Therefore, we believed that it would be possible to substantiate the benefit of prophylactic central neck dissection in hemithyroidectomy, while excluding other confounding factors.

Because hypocalcemia rarely occurs after hemithyroidectomy, this meta-analysis only analyzed clinical outcomes involving recurrence. In total, recurrence in the contralateral thyroid lobe was observed in $5.4 \%$ of patients, without a significant difference according to whether central neck dissection was performed, as expected. Recurrence in the lateral compartment was also not affected by central neck dissection, with an average recurrence rate of $1.31 \%$. However, the recurrence rate in the central compartment was significantly lower in patients who underwent prophylactic central neck dissection $(0.17 \%$ vs. $1.78 \%)$. This result is consistent with a previous meta-analysis investigating total thyroidectomy, and it clearly demonstrates the benefit of prophylactic central neck dissection without confounding factors. Although the benefit may be very small, we believe it is mean- 
ingful to minimize the recurrence rate, in that if recurrence is found later, patients can take medication for the rest of their lifetime after completion thyroidectomy with neck dissection. The side of recurrence in the central compartment has not been described in previous papers, with the exception of Hyun et al. [11], who described the side as unilateral. In conclusion, prophylactic central neck dissection during hemithyroidectomy for lowto intermediate-risk patients reduced the rate of recurrence in the central compartment by $1.6 \%$.

\section{CONFLICT OF INTEREST}

No potential conflict of interest relevant to this article was reported.

\section{ORCID}

Soon-Hyun Ahn https://orcid.org/0000-0002-0759-6850

Won Sik Kim https://orcid.org/0000-0002-9871-4655

\section{AUTHOR CONTRIBUTIONS}

Conceptualization: SHA. Data curation: all authors. Writing original draft: SHA.Writing - review \& editing: all authors.

\section{REFERENCES}

1. Mendelsohn AH, Elashoff DA, Abemayor E, St John MA. Surgery for papillary thyroid carcinoma: is lobectomy enough? Arch Otolaryngol Head Neck Surg. 2010 Nov;136(11):1055-61.

2. Barney BM, Hitchcock YJ, Sharma P, Shrieve DC, Tward JD. Overall and cause-specific survival for patients undergoing lobectomy, neartotal, or total thyroidectomy for differentiated thyroid cancer. Head Neck. 2011 May;33(5):645-9.

3. Koo BS, Choi EC, Yoon YH, Kim DH, Kim EH, Lim YC. Predictive factors for ipsilateral or contralateral central lymph node metastasis in unilateral papillary thyroid carcinoma. Ann Surg. 2009 May;249(5): 840-4.

4. Roh JL, Kim JM, Park CI. Central lymph node metastasis of unilateral papillary thyroid carcinoma: patterns and factors predictive of nodal metastasis, morbidity, and recurrence. Ann Surg Oncol. 2011 Aug;18(8):2245-50.

5. Hughes DT, Rosen JE, Evans DB, Grubbs E, Wang TS, Solorzano CC. Prophylactic central compartment neck dissection in papillary thyroid cancer and effect on locoregional recurrence. Ann Surg Oncol. 2018 Sep;25(9):2526-34.

6. Chen L, Wu YH, Lee CH, Chen HA, Loh EW,Tam KW. Prophylactic central neck dissection for papillary thyroid carcinoma with clinically uninvolved central neck lymph nodes: a systematic review and meta-analysis. World J Surg. 2018 Sep;42(9):2846-57.

7. Zhao WJ, Luo H, Zhou YM, Dai WY, Zhu JQ. Evaluating the effectiveness of prophylactic central neck dissection with total thyroidectomy for cNO papillary thyroid carcinoma: an updated meta-analy- sis. Eur J Surg Oncol. 2017 Nov;43(11):1989-2000.

8. Ebina A, Sugitani I, Fujimoto Y,Yamada K. Risk-adapted management of papillary thyroid carcinoma according to our own risk group classification system: is thyroid lobectomy the treatment of choice for low-risk patients? Surgery. 2014 Dec;156(6):1579-88.

9. Liu J, Zhang Z, Huang H, Xu S, Liu Y, Liu S, et al.Total thyroidectomy versus lobectomy for intermediate-risk papillary thyroid carcinoma: a single-institution matched-pair analysis. Oral Oncol. 2019 Mar;90: 17-22.

10. Choi JB, Lee SG, Kim MJ, Kim TH, Ban EJ, Lee CR, et al. Oncologic outcomes in patients with 1-cm to 4-cm differentiated thyroid carcinoma according to extent of thyroidectomy. Head Neck. 2019 Jan; 41(1):56-63.

11. Hyun SM, Song HY, Kim SY, Nam SY, Roh JL, Han MW, et al. Impact of combined prophylactic unilateral central neck dissection and hemithyroidectomy in patients with papillary thyroid microcarcinoma.Ann Surg Oncol. 2012 Feb;19(2):591-6.

12. Kuba S, Yamanouchi K, Hayashida N, Maeda S,AdachiT, Sakimura C, et al. Total thyroidectomy versus thyroid lobectomy for papillary thyroid cancer: comparative analysis after propensity score matching: a multicenter study. Int J Surg. 2017 Feb;38:143-8.

13. Kwon H, Jeon MJ, Kim WG, Park S, Kim M, Song DE, et al. A comparison of lobectomy and total thyroidectomy in patients with papillary thyroid microcarcinoma: a retrospective individual risk factormatched cohort study. Eur J Endocrinol. 2017 Apr;176(4):371-8.

14. Lee CR, Son H, Lee S, Kang SW, Jeong JJ, Nam KH, et al. Lobectomy and prophylactic central neck dissection for papillary thyroid microcarcinoma: do involved lymph nodes mandate completion thyroidectomy? World J Surg. 2014 Apr;38(4):872-7.

15. Li X, Zhao C, Hu D, Yu Y, Gao J, Zhao W, et al. Hemithyroidectomy increases the risk of disease recurrence in patients with ipsilateral multifocal papillary thyroid carcinoma. Oncol Lett. 2013 Apr;5(4): 1412-6.

16. Matsuzu K, Sugino K, Masudo K, Nagahama M, Kitagawa W, Shibuya $\mathrm{H}$, et al. Thyroid lobectomy for papillary thyroid cancer: long-term follow-up study of 1,088 cases. World J Surg. 2014 Jan;38(1):68-79.

17. Park YM, Lee DY, Oh KH, Cho JG, Baek SK, Kwon SY, et al. Clinical implications of pathologic factors after thyroid lobectomy in patients with papillary thyroid carcinoma. Oral Oncol. 2017 Dec;75:1-5.

18. Park JH, Lee YM, Lee YH, Hong SJ, Yoon JH. The prognostic value of serum thyroid-stimulating hormone level post-lobectomy in lowand intermediate-risk papillary thyroid carcinoma. J Surg Oncol. 2018 Sep;118(3):390-6.

19. Son HJ, Kim JK, Jung YD, Jang GH, Seo YT, Kim DS, et al. Comparison of outcomes between hemithyroidectomy alone and hemithyroidectomy with elective unilateral central neck dissection in patients with papillary thyroid microcarcinoma. Head Neck. 2018 Nov;40(11): 2449-54.

20. Song E, Han M, Oh HS, Kim WW, Jeon MJ, Lee YM, et al. Lobectomy is feasible for $1-4 \mathrm{~cm}$ papillary thyroid carcinomas: a 10-year propensity score matched-pair analysis on recurrence. Thyroid. 2019 Jan;29(1):64-70.

21. Vaisman F, Shaha A, Fish S, Michael Tuttle R. Initial therapy with either thyroid lobectomy or total thyroidectomy without radioactive iodine remnant ablation is associated with very low rates of structural disease recurrence in properly selected patients with differentiated thyroid cancer. Clin Endocrinol (Oxf). 2011 Jul;75(1):112-9.

22. Vaisman F, Momesso D, Bulzico DA, Pessoa CH, da Cruz MD, Dias F, et al.Thyroid lobectomy is associated with excellent clinical outcomes in properly selected differentiated thyroid cancer patients with primary tumors greater than $1 \mathrm{~cm}$. JThyroid Res. 2013;2013:398194.

23. Xue S, Wang P, Liu J, Chen G.Total thyroidectomy may be more reasonable as initial surgery in unilateral multifocal papillary thyroid microcarcinoma: a single-center experience. World J Surg Oncol. 
2017 Mar;15(1):62.

24. Haugen BR, Alexander EK, Bible KC, Doherty GM, Mandel SJ, Nikiforov YE, et al. 2015 American thyroid association management guidelines for adult patients with thyroid nodules and differentiated thyroid cancer: the American thyroid association guidelines task force on thyroid nodules and differentiated thyroid cancer. Thyroid. 2016 Jan;26(1):1-133.

25. Lang BH, Ng SH, Lau LL, Cowling BJ,Wong KP,Wan KY.A systematic review and meta-analysis of prophylactic central neck dissection on short-term locoregional recurrence in papillary thyroid carcinoma after total thyroidectomy. Thyroid. 2013 Sep;23(9):1087-98.

26. Ito Y, Miyauchi A, Kihara M, Higashiyama T, Kobayashi K, Miya A. Patient age is significantly related to the progression of papillary microcarcinoma of the thyroid under observation. Thyroid. 2014 Jan; 24(1):27-34.

27. Sugitani I, Fujimoto Y, Yamada K. Association between serum thyrotropin concentration and growth of asymptomatic papillary thyroid microcarcinoma. World J Surg. 2014 Mar;38(3):673-8. 